\title{
AITERIDAD Medios de comunicación organizacional interna en colegios públicos, Chile
}

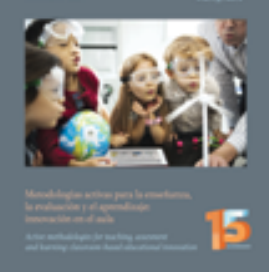

\author{
Methods of internal organizational communication in public \\ schools, Chile
}

Dra. Katia Papic-Domínguez es docente e investigadora de la Universidad Católica Silva Henríquez (Chile) (kpapic@ucsh.cl) (http://orcid.org/0000-0003-1091-4124)

Recibido: 2019-10-04 / Revisado: 2020-04-30 / Aceptado: 2020-06-08 / Publicado: 2020-07-01

\section{Resumen}

La civilización actual está fomentando innovaciones importantes en la gestión de los colegios públicos, donde la comunicación constituye una ocupación estratégica. El artículo tiene por objetivo describir los medios de comunicación organizacional interna utilizados mutuamente por el equipo de directivos y profesores, y su influencia en el robustecimiento del enlace cultural y en la gestión de la organización educativa municipalizada de los distritos de La Cisterna y Pudahuel, Santiago, Chile. Se utilizó una óptica mixta de naturaleza exploratoria y descriptiva, aplicada en una muestra de 22 instituciones educacionales municipalizadas: Para su fase cuantitativa se diseñó una encuesta que fue respondida por 246 personas; en su etapa cualitativa se utilizó una entrevista en profundidad aplicada a dos directores. Los resultados indican que los medios más utilizados por los participantes son las reuniones $(91,0 \%)$ y las circulares $(89,0 \%)$. Además, un $(89,0 \%)$ declara la presencia de valores organizacionales participados por los equipos directivos y profesorado. Puede concluirse como tendencia el empleo de medios de comunicación existentes en el colegio, a saber, interrelación personal, escrito, audiovisual, telecomunicación e informática destinados a transmitir información según las finalidades a lograr al remitirla; y, la inclinación de participar un léxico, valores organizacionales y convicciones. Se espera que los resultados generen un conocimiento que posibilite caracterizar las prácticas comunicativas en las organizaciones educativas e incrementar el acervo cultural en la materia.

Descriptores: Educación, gestión educativa, educación formal, organización educativa, Comunicación organizacional, colegios.

\section{Abstract}

The current civilization is promoting important innovations within the management of public schools, where communications it constitutes a strategic occupation. This article's objective to describe the methods of internal organizational communication used mutually by the team of managers and teachers, and their influence on the strengthening of the cultural link and on the management of the municipalized educational organization of the districts of La Cisterna and Pudahuel in Santiago, Chile. A mixed exploratory and descriptive approach was used, was applied to a sample of 22 municipal educational institutions: For the quantitative phase, a survey was designed and applied to 246 people, while in the qualitative phase, in-depth interviews were held with two school directors. The results indicate that the most common forms of communication used by the participants are meetings (91.0\%) and circulars (89.0\%). In addition, (89.0\%) declare the presence of organizational values participated by the management teams and teachers. It can be concluded as a trend the use of existing communication means in the school, that is, personal, written, audiovisual, telecommunication and computer interrelation destined to transmit information according to the purposes to be achieved when sending it; and, the inclination to participate in a lexicon, organizational values and convictions. These results are expected to generate knowledge that will enable a description of the communication practices within educational organizations and expand the culture body of evidence on this topic.

Keywords: Education, educational management, formal education, educational organization, organizational communication, teaching centers.

Forma sugerida de citar: Papic-Domínguez, K. (2020). Medios de comunicación organizacional interna en colegios públicos, Chile. Alteridad, 15(2), 241-255. https://doi.org/10.17163/alt.v15n2.2020.08 


\section{Introducción y estado de la cuestión}

En la civilización particularmente relevante es, según Martín (2000), el surgimiento del ecosistema comunicativo fundamentado en medios y tecnologías de comunicación e información, porque ello ha producido una motivación en el ámbito educativo (Aguaded, 2010). En tal sentido, Arboleda-Naranjo (2017) identifica la comunicación como la protagonista de los rápidos cambios que suceden en las organizaciones.

Frente a esta realidad, las organizaciones escolares consideran que la comunicación ejercita un papel relevante al constituir un soporte vital, para promocionar la consecución de las principales funciones de la organización (Villa et al., 2015), representando una influencia en su cultura (Palacios, 2015).

En Chile, en el sistema educativo nacional desde su marco regulatorio y marco curricular se promueve la mejora a través de la innovación tanto en la organización como en la gestión escolar, en especial se promociona el desarrollo de buenas prácticas en los colegios municipalizados, a modo de asegurar el cumplimiento de la educación con calidad, así como consolidar la educación pública escolar, con el objeto de superar su compleja situación (Ministerio de Educación de Chile, MINEDUC, 2015). Ello, requiere aplicar en acuerdo con Farfán-Tigre et al. (2016) una gestión focalizada en la calidad de las instituciones educativas porque conforma una demanda a atender en su mejora Así también, se necesita administrar la comunicación organizacional interna como una función estratégica asociada a la gestión del proyecto educativo institucional en la comunidad educativa. Porque la comunicación apunta Vizer (2018) se encuentra ligada de forma explícita o implícita a los procesos de entendimiento.

$\mathrm{Al}$ respecto, cabe pormenorizar que en el sistema escolar chileno se contemplan, en los niveles educativos de parvularia, de básica y de media, instituciones educacionales pagadas, que financian los padres o apoderados; y gratuitas, subvencionadas por el Estado. La educación pública escolar integra a los colegios municipalizados, objetos de la investigación, distinguiéndose como asimilados a Municipalidades para su administración y financiados por el Estado. Las municipalidades forman corporaciones autónomas de derecho público, encargada de administrar una comuna para dar satisfacción a las necesidades de la comunidad respectiva, en el ámbito social, de salud y de educación.

El presente artículo explora y describe los medios de comunicación organizacional interna utilizados al interior de los equipos de directores y los profesores, y su influencia en el robustecimiento del enlace cultural en la gestión educativa del colegio municipalizado, asimilado a dos distritos de la capital: La Cisterna y Pudahuel, Santiago, Chile. Se espera que sus resultados generen un conocimiento que posibilite caracterizar las prácticas comunicativas en las organizaciones educacionales de este tipo e incrementar el acervo cultural en la materia.

\subsection{Comunicación organizacional interna: Medios}

En la actualidad se constata que la comunicación organizacional interna se visualiza siguiendo a Cervera (2015) como una función consustancial de alguna actividad y se requiere administrarla y determinarla con precisión.

Desde este enfoque, la comunicación organizacional interna la conciben, Cuenca y Verazzi (2018), como una serie de acciones de comunicación realizadas por una organización, enfocadas a la creación y conservación de unas apropiadas relaciones con y al interior de sus miembros, mediante el uso de distintas técnicas comunicativas que los mantengan documentados, enlazados y motivados, aportando simultáneamente al logro de las finalidades organizacionales. También, la comunicación organizacional interna posibilita desplegar información global, dirigida a las audiencias internas así también enfocada a ampliar la cultura organizacional (Castillo, 2009). 
Para Villafañe (2002) los soportes de comunicación interna comprenden diversos medios y actuaciones para motivar, unir al recurso humano y establecer su aceptación de las metas organizacionales. De igual modo, Morató (2016) concibe al canal como un soporte, un medio para difundir información y un códice.

Lo anterior se complementa con lo señalado por Kaplún (1998), quien puntualiza que la utilización de ciertos medios de comunicación en la institución educativa debe ocurrir aplicando un enfoque crítico y reflexivo, como servicio y apoyo al proyecto pedagógico. Por ejemplo, en el medio escolar es posible identificar modelos de comunicación tradicionales, unidireccionales y escasos, con poco predominio de la interacción y de la participación, aunque se disponga de internet y de redes sociales que los favorecerían y potenciarían aún más (Palomares-Ruiz, 2015).

$\mathrm{Al}$ respecto, se aprecia como medios virtuales parafraseando a Enrique y Serrano (2015) la comunicación digital, las comunidades y las redes sociales. En ese sentido, internet puntualiza Túñez-López (2015) posibilita escuchar, dialogar y enlazarse directamente con los públicos y eso presupone una mutación de la cultura organizacional y del modo de gestionar sus procesos comunicacionales.

Por tanto, cabe apreciar que los medios escolares, explican Narváez-Montoya (2019), se enlazan con procedimientos comunicativos que integran el devenir organizacional, considerándola dimensión pública de la escuela, centrada en abordar las fases de interrelación y el papel de los medios disponibles en la escuela.

Al respecto, De Castro (2015) apunta medios de comunicación organizacional tales como reuniones, tablones de anuncios, periódico mural, correo electrónico y buzón de sugerencias, entre otros. Sin embargo, Macia (2019) explicita que los correos electrónicos y las plataformas digitales [...] constituyen medios, cuyo empleo está extendido insuficientemente.

Por último, en la institución la elección de canales de comunicación organizacional interna, según puntualiza Zapata (2016), se considera la utilización de los medios oficiales atendiendo sus rasgos distintivos y las finalidades a concretar.

\subsection{Organización educativa: Antecedentes para la gestión de la unión cultural}

En civilización actual, la organización y gestión escolar sugiere Batalloso (2017) deben sufrir transformaciones en las que se incentiven culturas profesionales de cooperación, coparticipación y compromiso porque una escuela [...] conforma una comunidad que comparte finalidades, experiencias y acontecer cotidiano. Por consiguiente, la función de comunicación organizacional interna es fundamental en el desenvolvimiento de una gestión escolar centrada en la innovación y mejora. En efecto, se estima que un líder adecuado siguiendo a Castilloveitía (2017) maneja con efectividad su comunicación.

Por su parte, el Gobierno de Chile (2017) define a los establecimientos educacionales, en la Ley 21.040 como la unidad esencial del sistema e integrada por comunidades educativas, que contemplan a los profesores, a los directivos, a los estudiantes, entre otros.

$\mathrm{Al}$ respecto, el Ministerio de Educación MINEDUC (2005) define la gestión escolar en términos de actividad experta y tecnológica, orientada a instaurar cualidades encauzadas a propiciar en los agentes el desenvolvimiento de buenas prácticas tendientes al logro de los objetivos institucionales. Para su implementación, se requiere desenvolver el robustecimiento de los procesos de comunicación ya que expresan Massoni et al. (2018) conforman una contribución al planeamiento y realización de las actividades de una organización. Aún más, se considera que las nuevas tecnologías según Lugo e Ithurburu (2019) generan una novedosa e imprescindible organización de los establecimientos educacionales.

Ahora bien, desde el despliegue de la gestión escolar se valora en la organización educativa 
el componente de la cultura, pues corresponde a la suma de convicciones, valores y normas de comportamiento, participadas y no transcritas, que utilizan los integrantes de la misma para regir sus actuaciones y, en cuanto a ello, se expresan a través de estas (Apolo et al., 2017).

Inclusive, cabe pensar de acuerdo a la Organización de las Naciones Unidas para la Educación, la Ciencia y la Cultura, UNESCO (2007) que la apreciación de la propia cultura conforma una condición fundamental para el desarrollo de una gestión educativa eficaz. Específicamente, la administración de la práctica de comunicación organizacional interna posibilita crear, desarrollar y asegurar el mantenimiento de la cultura en una organización social (Lacasa y Blay, 2004).

Por último, la estructura comunicacional y la gestión explica Pinto (2017) en la administración de una cultura participativa constituyen un componente sustantivo en tanto dispositivo teleológico tendiente al cumplimiento de los objetivos, creando huella en las entidades organizativas.

\section{Metodología}

La pregunta de investigación que se planteó en el estudio fue: ¿Cuáles son los medios comunicativos utilizados al interior del equipo de directivos y los docentes en la comunicación interna en pos de fomentar el robustecimiento de su enlace cultural en la gestión educativa de la institución educacional municipalizada, asimilada a los distritos de La Cisterna y Pudahuel, en Chile?

Para dar respuesta a ello se diseñó una investigación mixta, no experimental, sistemática y empírica (Hernández et al., 2014), así como también exploratoria, descriptiva y primaria, utilizando de forma conjunta el enfoque cuantitativo $y$ cualitativo.

Este estudio comprendió el empleo combinado del enfoque cuantitativo y cualitativo, porque Burke et al. (2007) la definen como una investigación en la que un investigador mezcla componentes de investigación cualitativa y cuantitativa, tales como empleo de perspectivas cualitativas y cuantitativas, recogida de datos, análisis e inferencia para concretar las finalidades de amplitud, profundidad, comprensión y corroboración. Además, se estimó adecuado emplear el método mixto, dada la complejidad del fenómeno en estudio. Por último, se valora la posibilidad de alcanzar un conocimiento más amplio y profundo sobre la realidad investigada, en consonancia con lo expuesto por Walton et al. (2019), porque señalan que el método mixto posibilita información sobre el fenómeno en estudio más holística y matizada.

Tanto en la etapa de diseño, de recogida de información como de análisis e interpretación se considera con el mismo nivel de importancia al enfoque cuantitativo y cualitativo, CUAN + CUAL. También, el diseño del estudio contempla la aplicación de ambos métodos de manera concurrente (simultánea) y de forma diligente, sistemática y empírica.

En la operacionalización del método mixto se contempló, principalmente, la determinación del sustento teórico conceptual de la comunicación organizacional interna, de la gestión y organización escolar junto con el enfoque mixto. Además, se planteó una pregunta de investigación principal amplia y compleja. En la recogida de datos se realizó la administración de los instrumentos cuantitativos y cualitativos de forma simultánea en las respectivas muestras. También, en lo sustantivo, el estudio analítico de los datos se concretó de forma sincrónica a través de programas computacionales. Por último, se elaboró un solo reporte, en donde se procedió a complementar los resultados, obtenidos desde la vertiente cuantitativa y cualitativa

\subsection{Etapa cuantitativa}

Esta fase consideró una población de 23 establecimientos educacionales municipalizados afiliados a las municipalidades de Pudahuel y La Cisterna, ubicados en el Área Metropolitana del país, dada la capacidad de acceso disponible. La 
muestra definitiva se determinó mediante un mecanismo probabilístico, con una población finita y baja, a la que se le administró un procedimiento aleatorio simple — de selección con tómbola- lo que derivó en una selección final de 22 establecimientos, de los cuales 15 correspondieron a la Municipalidad de Pudahuel y 7 a la Municipalidad de La Cisterna. La Tabla 1 describe y caracteriza los colegios municipalizados contemplados en la investigación.

Tabla 1. Caracterización de la población

\begin{tabular}{|l|c|l|c|c|c|}
\hline \multicolumn{1}{|c|}{ Comuna } & $\begin{array}{c}\mathbf{N}^{\circ} \text { establecimientos } \\
\text { educacionales }\end{array}$ & \multicolumn{1}{|c|}{ Niveles educativos } & $\begin{array}{c}\mathbf{N}^{\circ} \\
\text { directivos }\end{array}$ & $\begin{array}{c}\mathbf{N}^{\circ} \text { docentes } \\
\text { de aula }\end{array}$ & $\begin{array}{c}\mathbf{N}^{\circ} \\
\text { estudiantes }\end{array}$ \\
\hline Pudahuel & 16 & $\begin{array}{l}\text { Enseñanza media y/o bási- } \\
\text { ca y/o parvularia }\end{array}$ & 45 & 376 & 9.170 \\
\hline La Cisterna & 7 & $\begin{array}{l}\text { Enseñanza media y/o bási- } \\
\text { ca y/o parvularia }\end{array}$ & 22 & 110 & 1.879 \\
\hline Total final & 23 & $\begin{array}{l}\text { Enseñanza media y/o bási- } \\
\text { ca y/o parvularia }\end{array}$ & 67 & 486 & 11.049 \\
\hline
\end{tabular}

\section{Fuente: Elaboración propia.}

Para la definición de los establecimientos educacionales que conformarían la población de estudio se tuvieron en cuenta los principios siguientes:

- Constituir instituciones educacionales municipalizadas.

- Impartir enseñanza parvularia y/o enseñanza básica y/o enseñanza media.

- Ser administrados por el Director de Educación del distrito de Pudahuel o el Jefe del Departamento de Educación del distrito de La Cisterna.

- Estar ubicados geográficamente en el sector rural o urbano del distrito de La Cisterna y de Pudahuel.

- En funcionamiento en el año 2015.

- Capacidad de acceso para realizar la investigación.

Para la etapa de recogida de información se elaboró y utilizó una encuesta transversal o única de diseño propio, la cual se aplicó al $40,0 \%$ del equipo de directivos de cada establecimiento educacional municipalizado y al $40,0 \%$ del profesorado de cada uno de los 22 colegios. Asimismo, para complementar el criterio del
$40 \%$ se consideró necesario que, en el caso de los directivos, que estos fueran 2 de 3 o 3 de 4 por cada establecimiento educacional, dado que en las organizaciones educativas seleccionadas el número de directivos oscila entre 3 y 4 personas, por consiguiente, al aplicar este criterio, se garantizaba el cumplimiento de la recogida de información necesaria de la muestra. La encuesta se elaboró con un conglomerado de ítems, a partir de indicadores por dimensión y variables; además se determinó la codificación y la redacción de la totalidad de los elementos.

$\mathrm{El}$ instrumento integró preguntas de clase demográfica y de localización de la persona encuestada, tales como el puesto que desempeñaba en el colegio, así también consultas de estimación y de elección múltiple, las que consideraron una escala de proporción o razón contemplando categorías de respuesta tales como 1 (Muy en desacuerdo), 2 (En desacuerdo), 3 (Ni de acuerdo ni en desacuerdo), 4 (De acuerdo) y 5 (Muy de acuerdo). La codificación de los datos se efectuó junto con la confección de los ítems.

Del mismo modo, por medio del procedimiento de evaluación de jueces expertos se verificó la validez y confiabilidad del instrumento en cuanto a su contenido y, el alfa de Cronbach se 
aplicó para determinar la magnitud de coherencia interna. En tanto, se administró el instrumento de medición en una sola oportunidad sobre un total de nueve encuestas completadas, de manera de calcular el coeficiente, obteniendo un resultado de 0,93, lo que le otorga validez. Además, en la fase de evaluación y acendramiento del cuestionario se emplearon examinaciones de calidad del cuestionario, completitud, validez de códigos y consultas y, todo ello para disponer de un instrumento óptimo que posibilitara recopilar la mayor y mejor cantidad de respuestas esperadas.

En cuanto a las variables, las preguntas se distribuyeron de la siguiente forma:

\section{Tabla 2. Preguntas congregadas según sus variables, dimensiones e indicadores}

\begin{tabular}{|c|c|c|}
\hline Variables & Dimensiones & Indicadores \\
\hline $\begin{array}{l}\text { Empleo de múltiples medios } \\
\text { de comunicación interna al } \\
\text { interior de los equipos directi- } \\
\text { vos y los docentes. }\end{array}$ & $\begin{array}{l}\text { 1. Indicación de me- } \\
\text { dios comunicativos. } \\
\text { 2. Canales físicos. } \\
\text { 3. Soportes } \\
\text { personales. }\end{array}$ & $\begin{array}{l}\text { 1. Empleo de medios de comunicación de acuerdo } \\
\text { a la clase de lenguaje. } \\
\text { 2. Utilización de canales comunicativos en concor- } \\
\text { dancia a los propósitos por lograr. } \\
\text { 3. Indicación de canales de comunicación interna: } \\
\text { a. Circular. } \\
\text { b. Intranet. } \\
\text { c. Reunión. } \\
\text { d. Entrevista. } \\
\text { e. Tablón de avisos. } \\
\text { f. Círculos de calidad. } \\
\text { g. Informe anual de finanzas. } \\
\text { h. Reglamento interno. } \\
\text { i. Visitación al colegio. } \\
\text { j. Entorno virtual. } \\
\text { k. Publicación de prensa. } \\
\text { I. Buzón de sugerencias. } \\
\text { m. Videoconferencia. } \\
\text { n. Equipo de estudio. } \\
\text { o. Revista interna. } \\
\text { p. Otras. }\end{array}$ \\
\hline $\begin{array}{l}\text { El empleo fomenta el robus- } \\
\text { tecimiento del enlace cultural } \\
\text { en la gestión educativa de la } \\
\text { institución educacional. }\end{array}$ & $\begin{array}{l}\text { 1. Interrelación diaria } \\
\text { de la persona. } \\
\text { 2. Significación de } \\
\text { sucesos. }\end{array}$ & $\begin{array}{l}\text { 1. Valores de la institución. } \\
\text { 2. Reglamentación de la institución. } \\
\text { 3. Convicciones. } \\
\text { 4. Idearios. } \\
\text { 5. Léxico. }\end{array}$ \\
\hline
\end{tabular}

\section{Fuente: Elaboración propia.}

Tras la aplicación del instrumento a la muestra seleccionada de 22 establecimientos educacionales municipalizados, se recibieron 246 encuestas respondidas: 45 de ellas corres- pondientes a directivos (lo que representa el $67,0 \%$ del total de 67 integrantes de los equipos directivos, cifra señalada en la Tabla 1); y 201 pertenecientes al profesorado (es decir, el 41,0\% 
del total de 486 miembros del cuerpo de profe- la Tabla 3 describe la distribución por puesto sores indicado en la misma tabla). Por su parte, que ejercita de los informantes.

Tabla 3. Puesto que ejercita en la institución educativa

\begin{tabular}{|l|c|}
\hline Puesto & $\%$ \\
\hline Trabajador social & $0,4 \%$ \\
\hline Psicólogo & $0,9 \%$ \\
\hline Inspector & $1,8 \%$ \\
\hline Comisionado de convivencia & $2,7 \%$ \\
\hline Educador diferencial/Programa Integración Escolar & $4,0 \%$ \\
\hline Unidad Técnico Pedagógica & $4,4 \%$ \\
\hline Educador de párvulos & $6,2 \%$ \\
\hline Directivo & $6,6 \%$ \\
\hline Profesor & $73,0 \%$ \\
\hline Total & $100 \%$ \\
\hline
\end{tabular}

\section{Fuente: Elaboración propia.}

Por último, tras terminar la recogida de datos en el proceso cuantitativo se procedió a realizar la agrupación final, considerando para ello su correspondencia respecto de objetivos e hipótesis del estudio, las materias tratadas y las estrategias metodológicas aplicadas. De igual modo, el programa SPSS (IBM Corp., 2011) se usó para ejecutar el análisis de datos determinándose un libro de códigos con sus variables y los códigos correspondientes a las categorías en la matriz de datos, luego de ingresar los datos en ella se verificó la examinación estadística. Por su parte, el análisis contempló el grado de medición de cada variable y la estadística descriptiva integrada por la distribución de frecuencias y la moda como medida de tendencia central, su empleo permitió verificar tendencias y valorar los datos, a partir de estas pruebas se efectuó la interpretación, así como también en la exhibición de los datos se emplearon tablas y gráficos.

\subsection{Etapa cualitativa}

En esta fase de la recogida de información se contempló la selección de una muestra conformada por dos personas: El gerente del Departamento de Educación de La Cisterna y el gerente de la Dirección de Educación de Pudahuel. También, se consideró una muestra no probabilística, siguiendo a Hernández et al. (2006) denominada como casos de importancia para el objeto del estudiado así también políticamente relevantes. La determinación de la muestra respondió a los siguientes criterios:

- Selección mediante la estructura de Alta Dirección Pública aplicada a directivos de educación pública (municipal).

- Personeros que estuvieran desempeñando su cargo en el año 2015. 
Para este caso, se utilizó la técnica de recopilación de información identificada por Sierra Bravo (1994) como la entrevista abierta en profundidad transversal, administrada en investigaciones de clase exploratoria y descriptiva e indagaciones de carácter cualitativo, dado que posibilita adquirir información del informante con alto grado de especificidad, de diseño propio. Para esta etapa se elaboró una pauta de la entrevista con el objetivo de delinear sus alcances, de modo de recoger información acerca de diversos aspectos relativos a los canales de comunicación interna del equipo directivo y docentes en la realidad educacional. La guía de la entrevista estuvo integrada por preguntas de opinión y de conocimientos (Hernández et al., 2010).

Posterior a ello, se formuló una nómina de materias a estimar en las consultas, para posteriormente abordar el diseño del formato aplicando las definiciones técnicas. Las preguntas que contempló el instrumento fueron las siguientes:

- ¿Cuáles son los canales de comunicación interna de mayor empleo en el colegio por $\mathrm{y}$ al interior de docentes y equipo directivo? (pregunta de conocimiento).

- ¿Cómo el empleo de los soportes de comunicación organizacional interna favorece la conexión cultural entre el equipo directivo y profesores? (pregunta de opinión).

Finalmente, a través de la evaluación de especialistas se trató la validez interna cualitativa (Hernández et al., 2006) del instrumento, comprobando de esta manera su capacidad de comunicar el lenguaje, ideologías y ópticas de los sujetos entrevistados; así como su dependencia. Por ello, se proporcionó la pauta de la entrevista a cinco expertos, para que examinaran el contenido de las preguntas y verificaran si su diseño permitiría alcanzar el objetivo que se esperaba medir, cuyo resultado fue positivo.

Tras la recogida de datos se abordó el análisis cualitativo para generar los resultados, se realizó la incorporación de los datos al programa computacional, luego se efectuó un primer acercamiento exploratorio a los datos, además se organizaron atendiendo el puesto de trabajo, la asimilación al colegio, las variables y preguntas. Después, se concretó la examinación de los datos con el objeto de ordenarlos junto con darles significación conceptual, estableciendo coherencia respecto del fenómeno en estudio. La etapa de significación de sentido se verificó recurriendo al enfoque del investigador, quien revisó cada dato, identificando semejanzas, diferencias, significados, estructuras y tendencias. Para ello, cada entrevista abierta en profundidad - la realizada en el distrito de La Cisterna, con código 1 y la ejecutada en el distrito de Pudahuel, con código 2- fueron transcritas.

El análisis cualitativo se basó en la técnica de procesamiento de corte y clasificación; asimismo, se articuló en función de una guía integrada por propósito, que consideró unidades de análisis sobre las respuestas obtenidas, así como también las variables, las preguntas y subpreguntas, aspectos que luego fueron ordenados y presentados de acuerdo con el tópico de los resultados, utilizando los códigos previamente asignados.

\section{Resultados}

En cuanto al análisis cuantitativo, en la Tabla 4 se describe la variable, de la matriz, "Se emplean distintos medios de comunicación interna al interior de los equipos directivos y profesores", que corresponde a información recogida mediante la encuesta, en la fase cuantitativa. 
Tabla 4. Variable: Se emplean distintos medios de comunicación interna al interior de los equipos directivos y profesores. Secciones 1, 2 y 3 de la encuesta

\begin{tabular}{|c|c|c|c|c|c|}
\hline \multirow{2}{*}{\begin{tabular}{|c|c|c|} 
Categorías \\
Preguntas
\end{tabular}} & \multicolumn{5}{|c|}{ Frecuencias (porcentaje) } \\
\hline & 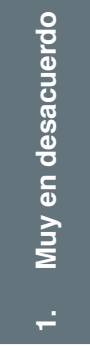 & 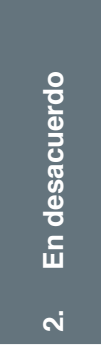 & 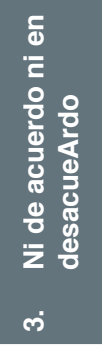 & 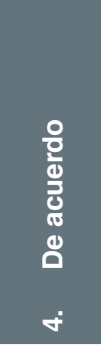 & 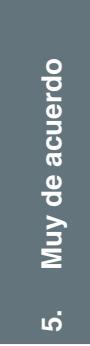 \\
\hline $\begin{array}{l}\text { 1. Usa los medios de comunicación existentes en la } \\
\text { institución educativa de acuerdo a la clase de len- } \\
\text { guaje no verbal o verbal empleado. }\end{array}$ & $1,2 \%$ & $1,6 \%$ & $8,6 \%$ & $48,2 \%$ & $40,4 \%$ \\
\hline $\begin{array}{l}\text { 2. Emplea canales de comunicación tales como inte- } \\
\text { rrelación personal, escrita, audiovisual, telecomu- } \\
\text { nicación e informático con la intención de transferir } \\
\text { información según las metas a alcanzar al difundirla. }\end{array}$ & $2,0 \%$ & $3,3 \%$ & $11,5 \%$ & $42,6 \%$ & $40,6 \%$ \\
\hline $\begin{array}{l}\text { 3. En la gestión de su colegio emplean canales perso- } \\
\text { nales a través de entregar información a otra, para } \\
\text { permitir la accesión a la información global de la en- } \\
\text { tidad organizativa. }\end{array}$ & $3,7 \%$ & $10,3 \%$ & $19,0 \%$ & $40,5 \%$ & $26,4 \%$ \\
\hline
\end{tabular}

\section{Fuente: Elaboración propia.}

Por otra parte, la Figura 1 resume cuáles de los encuestados respecto de las secciones $1 \mathrm{a}$ son los medios y dispositivos de comunicación 15, que corresponde a información recogida por organizacional interna de mayor empleo de parte medio de la encuesta, en la etapa cuantitativa.

Figura 1. Indique los medios y dispositivos que emplean en la comunicación organizacional interna. Secciones 1 a 15 de la encuesta

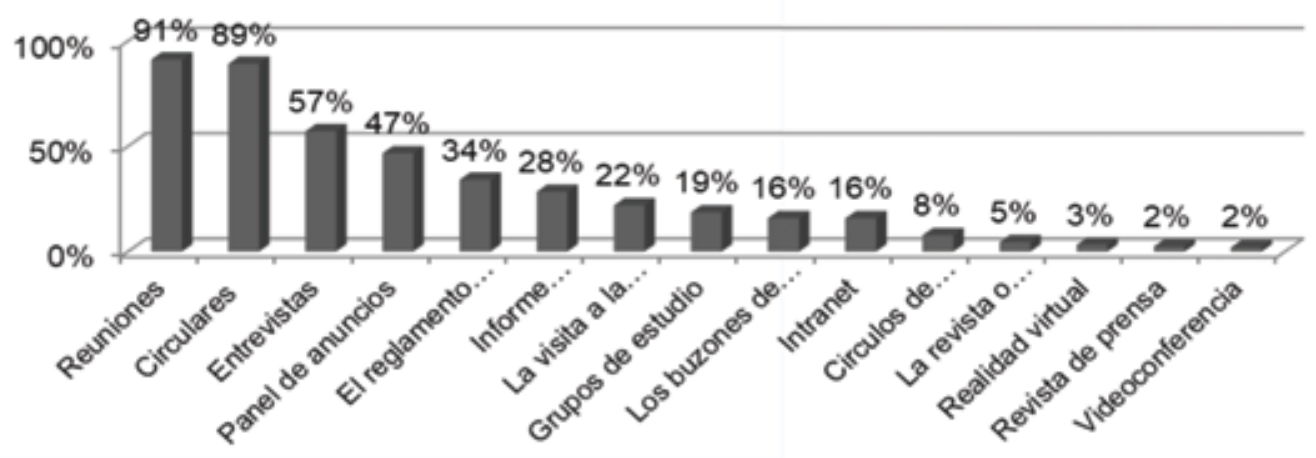

Fuente: Elaboración propia. 
Asimismo, los informantes, en la entrevista abierta en profundidad en la fase cualitativa, señalaron como medios fundamentales el correo electrónico (empleado para difundir memorándum y circulares) y el cuaderno de comunicación interna. Las reuniones también se utilizan de forma reiterada, las que generalmente son mensuales y donde participan equipos de gestión y profesionales. A nivel de comuna, la Dirección de Educación Municipal utiliza una página web para identificar y promocionar a los establecimientos educacionales municipalizados, además de la realización de visitas a las orga- nizaciones educativas en el transcurso del año. Complementariamente, en el último tiempo se organizó y comenzó a ser usado un grupo de WhatsApp entre los directores de las organizaciones educativas, para mantener una comunicación fluida. Complementa lo anterior el uso reducido de diarios murales.

La Figura 2 describe los medios y dispositivos empleados en la actividad comunicativa interna con sus indicadores y porcentajes, para la sección Otros, que corresponde a información recogida mediante la encuesta, en la fase cuantitativa.

Figura 2. Señale los canales y dispositivos empleados en la actividad comunicativa interna. Segmento 16 Otros, en la encuesta

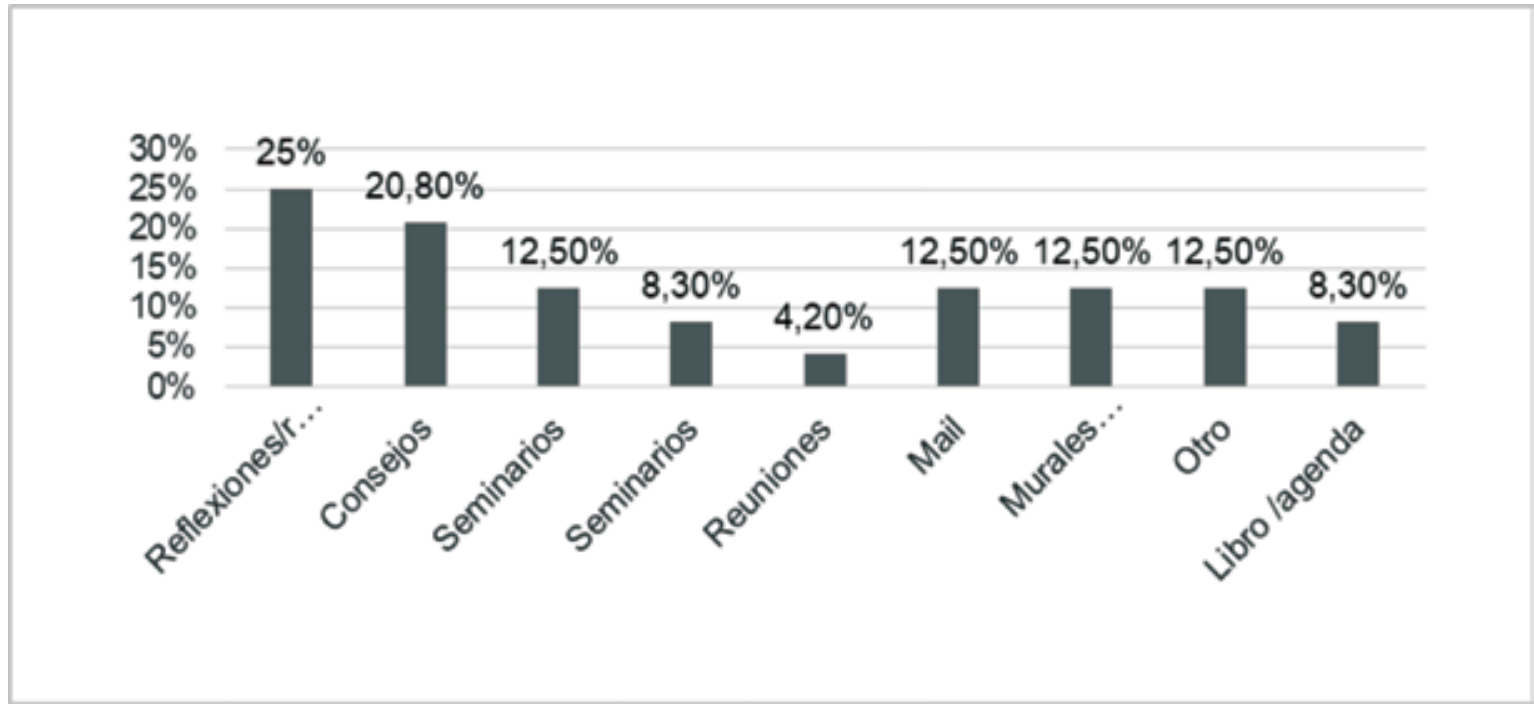

Fuente: Elaboración propia.

La Tabla 5 describe la variable de la matriz "Se incide en el robustecimiento del enlace cultural en la gestión escolar del colegio" que corresponde a información recogida a través de la encuesta, en la fase cuantitativa. 
Tabla 5. Variable: Se incide en el robustecimiento del enlace cultural en la gestión escolar del colegio. Secciones 4 a 9, de la encuesta

\begin{tabular}{|c|c|c|c|c|c|}
\hline \multirow{2}{*}{\begin{tabular}{|l} 
Categorias \\
Preguntas
\end{tabular}} & \multicolumn{5}{|c|}{ Frecuencia (porcentaje) } \\
\hline & 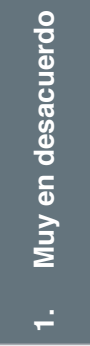 & 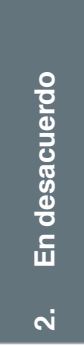 & 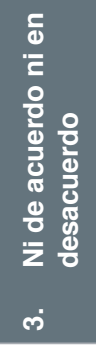 & 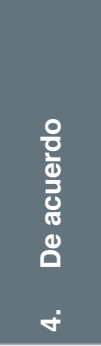 & 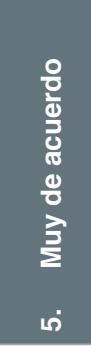 \\
\hline $\begin{array}{l}\text { 4. Las actividades comunicativas realizadas } \\
\text { por los profesores para los directivos les } \\
\text { permiten compartir el léxico. }\end{array}$ & $1,2 \%$ & $2,9 \%$ & $11,1 \%$ & $47,5 \%$ & $37,3 \%$ \\
\hline $\begin{array}{l}\text { 6. Usted observa la presencia de valores de } \\
\text { la institución participados por directivos y } \\
\text { profesores. }\end{array}$ & $1,6 \%$ & $2,0 \%$ & $7,3 \%$ & $45,7 \%$ & $43,3 \%$ \\
\hline $\begin{array}{l}\text { 6. En la interrelación personal de directores y } \\
\text { profesorado es posible distinguir los valo- } \\
\text { res de la institución. }\end{array}$ & $1,6 \%$ & $2,4 \%$ & $11,8 \%$ & $42,4 \%$ & $41,6 \%$ \\
\hline $\begin{array}{l}\text { 7. La relación interpersonal la regula apli- } \\
\text { cando la normativa de su organización } \\
\text { educativa. }\end{array}$ & $2,4 \%$ & $4,1 \%$ & $12,7 \%$ & $38,0 \%$ & $42,9 \%$ \\
\hline $\begin{array}{l}\text { 8. En las acciones tendientes a significar } \\
\text { los hechos del colegio utilizan un ideario } \\
\text { común. }\end{array}$ & $1,2 \%$ & $2,9 \%$ & $11,8 \%$ & $45,7 \%$ & $38,4 \%$ \\
\hline $\begin{array}{l}\text { 9. El desenvolvimiento de la interpretación de } \\
\text { los hechos del colegio incorpora el empleo } \\
\text { de convicciones compartidas. }\end{array}$ & $1,2 \%$ & $3,3 \%$ & $19,0 \%$ & $52,1 \%$ & $24,4 \%$ \\
\hline
\end{tabular}

\section{Fuente: Elaboración propia.}

Complementariamente, en la etapa cualitativa los entrevistados consignaron que el empleo de los medios de comunicación interna favorecía la unión cultural entre directivos y profesorado, al generarse un vínculo de dependencia y de pertenencia a la organización, a través de informar de un suceso o transmitir información.

En este mismo sentido, las jornadas de trabajo realizadas durante el año para tratar temas técnicos, dar informaciones y abordar la identidad cultural de los funcionarios con la institución educacional han posibilitado establecer un acompañamiento, brindar apoyo y establecer una asociación más estrecha entre el gerente de la Dirección de Educación Municipal junto con los equipos directivos de los establecimientos educacionales municipalizados.

Además, los entrevistados consignaron la necesidad de incrementar en el establecimiento educacional el empleo de medios y dispositivos de comunicación organizacional interna, porque se comparte en el colegio una cultura colaborativa de trabajo con códigos de expresión definidos por las jefaturas y profesorado. Así mismo, se señaló la existencia de una cultura de carácter punitiva, producida por los establecimientos educacionales municipales, los organismos ministeriales, la Superintendencia de Educación, 
la Agencia de Calidad de la Educación y la Dirección de Educación Municipal, motivada por sus funciones de supervisión, control e intervenciones, temas en que sería necesario contar con mayores y mejores mecanismos de comunicación interna.

\section{Discusión y conclusiones}

\subsection{Discusión}

Se aprecia una disposición, por parte de directivos y profesorado, de contar con un sistema comunicativo que contemple una variedad de medios de comunicación organizacional interna y utilizarlos para transmitir y compartir información. Esta condición coincide con Castillo (2009) porque afirma que la comunicación interna posibilita desplegar una información general.

Se observa la aplicación de una estrategia comunicativa por los directivos dirigida al profesorado para promover la motivación, la unión y la transmisión de información mediante la utilización habitual de medios como reuniones, circulares, entrevistas, correo electrónico y memorándum, entre otros. Lo anterior coincide con Cuenca y Verazzi (2018), quienes indican que la comunicación interna comprende una serie de acciones comunicativas realizadas por una organización, centradas en la creación y conservación de adecuadas interacciones con y al interior de sus miembros, mediante la utilización de técnicas comunicativas para estar informados, unidos y motivados, contribuyendo simultáneamente al cumplimiento de los objetivos organizacionales.

Ahora bien, en relación con la Figura 2, a pesar de la trascendencia de desarrollar la mejora de la calidad educativa, los participantes solo declararon realizar reflexiones pedagógicas $(25,0 \%)$ y consejos $(20,8 \%)$, lo que podría aludir a un trabajo colectivo del profesorado y los directivos, focalizado en temas administrativos, más que en gestión técnico-pedagógica.

Estas cifras en el sistema y estrategia comunicativa interna son muy descendidas res- pecto del empleo de reuniones (con un 91,0\%); circulares (con un 89,0\%); y entrevistas (con un $57,0 \%$ ); situación que atañe a un estilo directivo y de liderazgo en la gestión escolar que conlleva al fortalecimiento de su cultura escolar, sobre la base de actuaciones fundamentadas en valores, ideas, entre otros, compartidas por directivos y profesorado. Ello, coincide con Apolo et al. (2017) en el sentido de que la cultura comprende la suma de convicciones, valores y normas de conducta que utilizan los integrantes de una organización para regir sus actuaciones $\mathrm{y}$, por tanto, se manifiestan mediante estas.

El desenvolvimiento de la gestión de la cultura en el funcionamiento cotidiano constituye una condición que fortalece las relaciones comunicativas establecidas por directivos y profesores, porque coadyuvan a instalar valores institucionales compartidos, motivación y cohesión comunitaria, favoreciendo así el cumplimiento de las intenciones académicas y administrativas en la institución educacional, lo cual es coherente con lo planteado por MINEDUC (2005) en cuanto a que la gestión escolar está dirigida a la creación de condiciones que favorezcan dichos procesos.

Por otra parte, la vivencia de los valores organizacionales se institucionaliza en la práctica educativa desde la relación interpersonal dada entre los directivos y profesorado y, por ende, la interacción se realiza de manera conjunta y simultáneamente con la actividad comunicativa, empleando las instancias y los medios disponibles en la organización. Por ello, se requiere según Massoni et al. (2018) robustecer los procesos de comunicación para contribuir al planeamiento y despliegue de las acciones de una entidad organizativa.

En la perspectiva de la gestión directiva se contempla la práctica de la reflexión crítica docente en donde, mediante los medios de comunicación, se comparten ideas, principios y conceptos para establecer perspectivas consensuadas en torno a las condiciones de la organización educativa, constituyéndose de esta manera en un factor clave del desarrollo y fortalecimiento de la 
toma de conocimiento, implicación y vivencia de la cultura. En tal sentido, la administración de la comunicación organizacional interna posibilita crear, desarrollar y asegurar el mantenimiento de la cultura en una organización social (Lacasa y Blay, 2004).

\subsection{Conclusiones}

La investigación permitió identificar una predisposición entre los directivos y el profesorado participantes del estudio sobre el empleo de los medios y dispositivos de comunicación existentes en sus establecimientos, a saber las relaciones interpersonales, manuscritos, audiovisuales, telecomunicaciones e informáticos, usados para difundir informaciones en concordancia con los propósitos de comunicación, así como también el uso de medios personales para acceder a la información global del colegio, dado que ello les posibilita conocerse, unirse y motivarse en la dimensión laboral y relacional. Al respecto, el estudio reveló que los directivos y el profesorado utilizan mayoritariamente las reuniones (un $91,0 \%$ ) y las circulares (un 89,0\%) como instancias de información, focalizando en estos elementos su estrategia comunicativa, lo que evidencia la necesidad de ampliar la variedad de medios de comunicación organizacional interna y cumplir su uso para enfrentar adecuadamente los desafíos y requerimientos de actualización de los modos de gestionar en la organización educativa.

Asimismo, se reconoce una inclinación en el enlace cultural de los agentes participantes, porque se distingue al interior de los directores y docentes actividades que conllevan un léxico común, ciertos valores organizacionales, algunas convicciones e ideas que les posibilitan significar los acontecimientos organizacionales. También, se acepta que los canales de comunicación organizacional interna fomentan cierta creación, así como conservación de vínculos de afiliación y supeditación al establecimiento como una identidad cultural. En la actualidad se le demanda al establecimiento educacional municipalizado incorporar organizacionalmente y en el aula estos nuevos rasgos culturales, rol que le compete a los directivos y al profesorado, como un fundamento para actualizar los modos de gestionar ambos espacios, debiendo emplear para su concreción la comunicación organizacional interna.

Los resultados de este estudio son importantes de considerar en el escenario chileno actual, donde se visualiza el requerimiento de potenciar el perfeccionamiento en la gestión educativa de las instituciones educacionales municipalizadas (públicas) con la finalidad de desarrollar la educación de calidad. Para ello, se requiere fortalecer una cultura colaborativa mediante la pesquisa de innovaciones comunicativas orientadas a diversificar los medios de comunicación organizacional, a planificar estrategias comunicacionales y a examinar los procesos comunicativos para beneficiar la difusión de la información, el interés, el compromiso junto con la unión de profesores, de directores $y$, en conjunto, con el objeto de avanzar hacia el robustecimiento de la calidad educativa de las instituciones municipalizadas (pública). Finalmente, se sugieren las líneas de investigación de la comunicación organizacional, de la cultura y comunicación y del marketing.

\section{Referencias bibliográficas}

Aguaded, I. (2010). La educación para la comunicación. Nueva alfabetización para un mundo global. Octaedro.

Apolo, D., Báez, V., Pauker, L., \& Pasquel, G. (2017). Gestión de comunicación corporativa: Consideraciones para el abordaje de su estudio y práctica. [Corporate Communication Management:Considerations for the approach to its study and practice]. Revista Latina de Comunicación Social, (72), 521-539. https://doi.org/ 10.4185/RLCS-2017-1177

Arboleda-Naranjo, R. (2017). Estructuras disipativas en la comunicación y el comportamiento organizacional. [Dissipative Structures in Communication and Organizational Behavior]. Revista Razón y Palabra, 21(297), 498-521. https://bit.ly/2qfcHhd 
Batalloso, J. (2017). Paisajes educativos y escenarios escolares. Bubok Publishing S.L. https://bit.ly/3hgV9Hp

Burke, R., Onwuegbuzie, A., \& Turner, L. (2007). Toward a Definition of Mixed Methods Research. Journal of Mixed Methods Research, (1)2, 112-133. https://doi.org/10.1177/1558689806298224

Castillo, A. (2009). Relaciones públicas. Teoría e historia. Editorial UOC.

Castilloveitía, A. (2017). Las destrezas de comunicación del director escolar y el manejo de conflicto en Puerto Rico. [The School Principal's Communication Skills and Conflict Management in Puerto Rico]. Revista Iberoamericana sobre Calidad, Eficacia $y$ Cambio en Educación, 15(3), 113-128. https://doi.org/10.15366/reice2017.15.3.006

Cervera, Á. (2015). Comunicación total. Quinta edición. ESIC Editorial.

Cuenca, J., \& Verazzi, L. (2018). Guía fundamental de la comunicación interna. Editorial UOC.

De Castro, A. (2015). Manual práctico de comunicación organizacional. Serie de manuales prácticos. Editorial Universidad del Norte.

Enrique, J. \& Serrano, M.F. (Eds.). (2015). Somos estrategas: Dirección de comunicación empresarial e institucional. Editorial Gedisa.

Farfán-Tigre, Á., Mero-Delgado, O., \& SáenzGavilanes, J., (2016). Consideraciones generales acerca de la gestión educativa. [General considerations about educational management]. Revista Científica Dominio de las Ciencias, (2) 4, 179-190. https://bit.ly/2qfoxbc

Gobierno de Chile (2017), Ley Crea el Sistema de Educación Pública $\mathrm{N}^{\circ} 21.040$, de 24 de noviembre, de Educación. Ministerio de Educación.

Hernández, R., Fernández-Collado, C., \& Baptista, P. (2006). Metodología de la investigación. McGraw-Hill Interamericana Editores S.A.

Hernández, R., Fernández-Collado, C., \& Baptista, P. (2014). Metodología de la investigación. Sexta edición. McGraw-Hill Interamericana Editores S.A. https://bit.ly/2CaImmQ

IBM Corp. (2011). IBM SPSS Statistics for Windows, Version 20.0. IBM Corp.

Kaplún, M. (1998). Procesos educativos y canales de comunicación. [Educational processes and channels of communication]. Chasqui, 64,48. https://bit.ly/36vqH79

Lacasa y Blay, A. (2004). Gestión de la comunicación empresarial. Ediciones Gestión 2000.

Lugo, M. \& Ithurburu, V. (2019). Políticas digitales en América Latina. Tecnologías para fortalecer la educación de calidad. [Digital policies in Latin America. Technologies to improve quality education]. Revista Iberoamericana De Educación, 79(1), 11-31.

https://doi.org/https://doi.org/10.35362/rie7913398

Macia, M. (2019). Principales canales para la comunicación familia-escuela: Análisis de necesidades y propuestas de mejora. [Main channels for family-school communication: Needs analysis and proposals for improvement]. Revista Complutense de Educación, 30(1), 147-165. https://doi.org/10.5209/ RCED.56034

Martín, J. (2000). Retos culturales: De la comunicación a la educación. [Cultural challenges from communication to education]. Nueva Sociedad, 169, 33-43. https://bit.ly/2NaNRYQ Massoni, S., Mascotti, M., \& Margherit, L. (2018). Medición de procesos comunicacionales en organizaciones y proyectos: La dimensión informativa desde la comunicación estratégica. [Measurement of Communication Processes in Organizations and Projects: The Information Dimension from Strategic Communication] Razón y Palabra, 22(1100), 441-460. https://bit.ly/32aXrPU

Ministerio de Educación de Chile, Mineduc (2005). Sistema de Aseguramiento de la Calidad de la gestión Escolar. Calidad en todas las escuelas y liceos. Ministerio de Educación.

Ministerio de Educación de Chile, Mineduc (2015). Mapa de la reforma educacional. https://bit.ly/2NDbVm5

Morató, B. (2016). La comunicación corporativa. https://bit.ly/2Yb8gkI

Narváez-Montoya, A. (2019). Comunicación educativa, educomunicación y educación mediática: Una propuesta de investigación y formación desde un enfoque culturalista. [Educational Communication, Educommunication, and Media Education: A Research and Educational Proposal from a Culturalist Approach], Palabra Clave, 22(3), 1-30. 
http://doi.org/10.5294/pacla.2019.22.3.11

Organización de las Naciones Unidas para la Educación, la Ciencia y la Cultura (UNESCO) (2007). De la administración escolar tradicional a la gestión educativa estratégica. https://bit.ly/2PKcgpO

Palacios, J. (2015). Historia y avances en la investigación en comunicación organizacional. [History and advances in communication organizational research]. Revista Internacional de Relaciones Públicas, V(10), 25-46. http://dx.doi.org/10.5783/RIRP-10-2015-03-25-46

Palomares, A. (2015). Análisis de modelos de comunicación, profesorado-familia, para gestionar conflictos: Estudio de la comunidad educativa de Albacete. [Teachers-family communication models analysis to manage conflict: Study of albacete's educational communityn] Pedagogía Social. Revista Interuniversitaria, 25, 277-298. https://doi.org/10.7179/PSRI_2015.25.12

Pinto, A. (2017). El sistema comunicacional y su incidencia en la gestión de la investigación desarrollada en la Universidad Técnica de Babahoyo, provincia de Los Ríos, Ecuador. https://bit.ly/3fa08Ib

Sierra, R. (1994). Tesis doctorales y trabajos de investigación científica. Paraninfo S.A.

Túñez-López, M. (2015). Editorial: Modelo de simetría interactiva en Comunicación organizacional. Revista Mediterránea de Comunicación, 6(2), 5-7.

https:// doi.org/ 10.14198/MEDCOM2015.6.2.14

Villa, A., Troncoso, P., \& Díez, F. (2015). Estructura latente y fiabilidad de las dimensiones que explican el impacto de los sistemas de gestión de calidad en los centros educativos. [Latent structure and reliability of dimensions that explain the impact of quality management systems on schools]. Enseñanza, 33(1), 65-82. https://doi.org/10.6018/rie.33.1.199921

Villafañe, J. (2002). Imagen positiva. Gestión estratégica de la imagen de las empresas. Pirámide.

Vizer, E. (2018). Notas para una ontología de la comunicación II: Sobre las Materialidades de la Comunicación. [Notes for an ontology of communication II: About the "materialities of communication]. Palabra Clave, 21(2), 553-592. https://doi.org/10.5294/pacla.2018.21.2.12

Walton, J., Plano-Clark, V, Foote, L., \& Johnson, C. (2019). Navigating Intersecting Roads in a Mixed Methods Case Study: A Dissertation Journey. Journal of Mixed Methods Research, (1) 20. https://doi.org/10.1177/1558689819872422

Zapata, L. (2016). Industria de la comunicación y economía digital: Guía básica del Dircom. Editorial UOC. 